\title{
The solution of elasticity problems for the half-space by the method of Green and Collins
}

\author{
J.R. BARBER \\ Department of Mechanical Engineering and Applied Mechanics, The University of Michi- \\ gan, Ann Arbor, MI 48109, USA
}

\begin{abstract}
The paper reviews the method of complex potential functions developed by Green and Collins as applied to axisymmetric mixed boundary value problems in elasticity for the half-space. It is shown how the method can be applied to problems in several coupled potential functions such as adhesive and frictional contact problems, to problems involving annular regions and to problems in thermoelasticity. Attention is given to the question of choosing a formulation which leads to a well-behaved numerical solution.

Tables are given of the most commonly needed inversion formulae and of expressions for total load and stress intensity factor.
\end{abstract}

\section{Introduction}

The author has recently solved a number of axisymmetric mixed boundary value problems $[1-5]$ in elasticity using the harmonic potential function representation developed by Green and Collins. This method is less popular than the Hankel transform representation pioneered by Sneddon [6,7], but it has two significant advantages: (i) it leads directly to an integral equation formulation and (ii) the representations can be chosen to have the correct asymptotic behavior at the edges of the several regions so that the resulting integral equations are well suited to a rapidly convergent numerical solution.

The two methods are of course very closely related and can also be used in combination. A number of interesting and illuminating interrelationships are given by Keer [8], Sneddon [6] and Gladwell [9].

In this paper, we shall discuss the method in more detail and generality than could be justified in the solution of a particular problem and record various results and relationships which facilitate the use of the method. 


\section{Historical background}

The method can be traced back to Love's solution [10] of the Boussinesq frictionless punch problem. He showed that a series of complex harmonic potential functions can be generated from real Legendre polynomial solutions in cylindrical coordinates $(r, \theta, z)$ by substituting $(z+i a)$ for $z$. The real and imaginary parts of the resulting functions are separately harmonic and have discontinuities at $r=a$ on the plane $z=0$.

Functions of this type can be used in combination with the Papcovich-Neuber or the Green-Zerna potentials for the equations of elasticity to give elegant solutions to the classical frictionless punch and crack problems. Several examples are treated by Green [11], who uses Copson's solution in terms of Abel integrals [12] to obtain the complete surface values of the potentials, but uses Love's method semi-intuitively to extend these results to the rest of the half-space.

Green and Zerna [13] extended the method to give a general solution for the axisymmetric frictionless punch using the integral representation

$$
\phi=R \int_{0}^{a} \frac{g(t) \mathrm{d} t}{\left(r^{2}+(z+i t)^{2}\right)^{1 / 2}}=\frac{1}{2} \int_{-a}^{+a} \frac{g(t) \mathrm{d} t}{\left(r^{2}+(z+i t)^{2}\right)^{1 / 2}}
$$

where $g(t)$ is an even function of $t$. It can be shown that Eq. (1) satisfies identically the condition $\partial \phi / \partial z=0, r>a, z=0$ and the remaining boundary condition in $0 \leq r \leq a, z=0$ will yield an Abel integral equation for the unknown function $g(t)$.

In effect, Eq. (1) is a superposition of solutions of the form $R\left(r^{2}+(z+i t)^{2}\right)^{-1 / 2}$ derived from the 'point source' solution $\left(r^{2}+z^{2}\right)^{-1 / 2}$. We could therefore describe $\phi$ as the potential due to an arbitrary distribution $g(t)$ of point sources along the imaginary $z$ axis between $z=0$ and $z=i a$. It is therefore a logical development of the classical method of obtaining axisymmetric potential functions by distributing singularities along the axis of symmetry. A closely related solution is given by Segedin [14] who develops it as a convolution integral of an arbitrary kernel function with the classical potential solution of Boussinesq. He uses this method to obtain the solution for a power law punch $\left(u_{\mathrm{z}} \sim r^{n}\right)$ and treats more general problems by superposition after expanding the punch as a power series in $r$. It should be noted that Segedin's solution is restricted to indentation by a punch of continuous profile, in which case the contact radius $a$ is not known a priori. In practice, $a$ can be prescribed provided the rigid body indentation of the punch is allowed to float. The idea of representing a general solution by superposition of Boussinesq type solutions for different values of the radius has also been used as a direct numerical method by Maw et al. [15].

Green's method was extensively developed by Collins who used it to 
treat many interesting problems including the indentation problem for an annular punch [16] and a problem with radiation boundary conditions [17].

We should also note the significant contribution of $\operatorname{Keer}[8,18,19]$ who extended the integral representation to include non-axisymmetric problems, and the application to thermoelastic problems involving two or more potential functions by Shail [20] and the present author [1-5].

\section{Basic forms and surface values}

For axisymmetric elasticity problems in the half-space, a convenient representation of the displacement $\mathbf{u}$ can be obtained from Green and Zerna potentials [13] as

$$
\begin{aligned}
\mathbf{u}= & \frac{(1-\nu)}{\mu} \nabla \psi_{1}+\frac{z}{2 \mu} \nabla \frac{\partial \psi_{1}}{\partial z}-\frac{(3-4 \nu)}{2 \mu} \mathbf{k} \frac{\partial \psi_{1}}{\partial z} \\
& +\frac{(1-2 \nu)}{2 \mu} \nabla \psi_{2}+\frac{z}{2 \mu} \nabla \frac{\partial \psi_{2}}{\partial z}-\frac{(3-4 \nu)}{2 \mu} \mathbf{k} \frac{\partial \psi_{2}}{\partial z}
\end{aligned}
$$

where $\psi_{1}, \psi_{2}$ are axisymmetric harmonic functions.

At the surface, $z=0$, the stress and displacement components reduce to

$$
\left.\begin{array}{l}
\sigma_{r z}=\frac{\partial^{2} \psi_{1}}{\partial r \partial z} ; \quad \sigma_{z z}=-\frac{\partial^{2} \psi_{2}}{\partial z^{2}} \\
u_{r}=\frac{(1-\nu)}{\mu} \frac{\partial \psi_{1}}{\partial r}+\frac{(1-2 \nu)}{2 \mu} \frac{\partial \psi_{2}}{\partial r} \\
u_{z}=-\frac{(1-2 \nu)}{2 \mu} \frac{\partial \psi_{1}}{\partial z}-\frac{(1-\nu)}{\mu} \frac{\partial \psi_{2}}{\partial z}
\end{array}\right\}
$$

To represent the potential functions $\psi_{1}, \psi_{2}$ we use suitable combinations of the four basic forms

$$
\left.\begin{array}{l}
\phi_{1}=R \int_{0}^{a} g_{1}(t) F(r, z, t) \mathrm{d} t \\
\phi_{2}=R \int_{a}^{\infty} g_{2}(t) F(r, z, t) \mathrm{d} t \\
\phi_{3}=\operatorname{Im} \int_{0}^{a} g_{3}(t) F(r, z, t) \mathrm{d} t \\
\phi_{4}=\operatorname{Im} \int_{a}^{\infty} g_{4}(t) F(r, z, t) \mathrm{d} t
\end{array}\right\}
$$

where

$$
F(r, z, t)=\log \left(\left(r^{2}+(z+i t)^{2}\right)^{1 / 2}+(z+i t)\right)
$$


is a complex harmonic function obtained on the pattern of Eq. (1) from the real potential function $\log \left(\sqrt{r^{2}+z^{2}}+z\right)$.

The square root in (6) is interpreted as

$$
\left(r^{2}+(z+i t)^{2}\right)^{1 / 2}=\rho \mathrm{e}^{i v / 2}
$$

where

$$
\rho \geq 0 ; \quad 0 \leq v<\pi .
$$

\begin{tabular}{|c|c|c|}
\hline & $0<r<a$ & $r>a$ \\
\hline$\partial \phi_{1} / \partial r$ & $\frac{1}{r} \int_{0}^{a} g_{1}(t) \mathrm{d} t-\frac{1}{r} \int_{r}^{a} \frac{t g_{1}(t) \mathrm{d} t}{\sqrt{t^{2}-r^{2}}}$ & $\frac{1}{r} \int_{0}^{a} g_{1}(t) \mathrm{d} t$ \\
\hline$\partial \phi_{1} / \partial z$ & $\int_{0}^{r} \frac{g_{1}(t) d t}{\sqrt{r^{2}-t^{2}}}$ & $\int_{0}^{a} \frac{g_{1}(t) \mathrm{d} t}{\sqrt{r^{2}-t^{2}}}$ \\
\hline$\partial^{2} \phi_{1} / \partial z^{2}$ & $\frac{1}{r} \mathrm{~d} / \mathrm{d} r \int_{r}^{a} \frac{t g_{1}(t) \mathrm{d} t}{\sqrt{t^{2}-r^{2}}}$ & 0 \\
\hline$\partial \phi_{2} / \partial r$ & $\frac{1}{r} \int_{a}^{\infty} g_{2}(t) \mathrm{d} t-\frac{1}{r} \int_{a}^{\infty} \frac{t g_{2}(t) \mathrm{d} t}{\sqrt{t^{2}-r^{2}}}$ & $\frac{1}{r} \int_{a}^{\infty} g_{2}(t) \mathrm{d} t-\frac{1}{r} \int_{r}^{\infty} \frac{t g_{2}(t) \mathrm{d} t}{\sqrt{t^{2}-r^{2}}}$ \\
\hline$\partial \phi_{2} / \partial z$ & 0 & $\int_{a}^{r} \frac{g_{2}(t) \mathrm{d} t}{\sqrt{r^{2}-t^{2}}}$ \\
\hline$\partial^{2} \phi_{2} / \partial z^{2}$ & $\frac{1}{r} \mathrm{~d} / \mathrm{d} r \int_{a}^{\infty} \frac{\operatorname{tg}_{2}(t) \mathrm{d} t}{\sqrt{t^{2}-r^{2}}}$ & $\frac{1}{r} \mathrm{~d} / \mathrm{d} r \int_{r}^{\infty} \frac{\operatorname{tg}(t) \mathrm{d} t}{\sqrt{t^{2}-r^{2}}}$ \\
\hline$\partial \phi_{3} / \partial r$ & $-\frac{1}{r} \int_{0}^{r} \frac{\operatorname{tg}_{3}(t) \mathrm{d} t}{\sqrt{r^{2}-t^{2}}}$ & $-\frac{1}{r} \int_{0}^{a} \frac{\operatorname{tg}_{3}(t) \mathrm{d} t}{\sqrt{r^{2}-t^{2}}}$ \\
\hline$\partial \phi_{3} / \partial z$ & $-\int_{r}^{a} \frac{g_{3}(t) \mathrm{d} t}{\sqrt{t^{2}-r^{2}}}$ & 0 \\
\hline$\partial^{2} \phi_{3} / \partial z^{2}$ & $\frac{1}{r} \mathrm{~d} / \mathrm{d} r \int_{0}^{r} \frac{\operatorname{tg}_{3}(t) \mathrm{d} t}{\sqrt{r^{2}-t^{2}}}$ & $\frac{1}{r} \mathrm{~d} / \mathrm{d} r \int_{0}^{a} \frac{\operatorname{tg}_{3}(t) \mathrm{d} t}{\sqrt{r^{2}-t^{2}}}$ \\
\hline$\partial \phi_{4} / \partial r$ & 0 & $-\frac{1}{r} \int_{a}^{r} \frac{t g_{4}(t) \mathrm{d} t}{\sqrt{r^{2}-t^{2}}}$ \\
\hline$\partial \phi_{4} / \partial z$ & $-\int_{a}^{\infty} \frac{g_{4}(t) \mathrm{d} t}{\sqrt{t^{2}-r^{2}}}$ & $-\int_{r}^{\infty} \frac{g_{4}(t) d t}{\sqrt{t^{2}-r^{2}}}$ \\
\hline$\partial^{2} \phi_{4} / \partial z^{2}$ & 0 & $\frac{1}{r} \mathrm{~d} / \mathrm{d} r \int_{a}^{r} \frac{t g_{4}(t) \mathrm{d} t}{\sqrt{r^{2}-t^{2}}}$ \\
\hline
\end{tabular}

Table 1. Surface values of derivatives of the functions $\phi_{i}$ (Eqs. (5)) 
Equations (5) can be written in two alternative forms which for $\phi_{1}$ are

$$
\phi_{1}=\frac{1}{2} \int_{0}^{a} g_{1}(t)[F(z, r, t)+F(z, r,-t)] \mathrm{d} t
$$

and

$$
\phi_{1}=\frac{1}{2} \int_{-a}^{+a} g_{1}(t) F(z, r, t) \mathrm{d} t .
$$

We note that (10) is exactly equivalent to (5) if and only if $g_{1}$ is an even function of $t$. If the boundary values of $\phi, \partial \phi / \partial z, \partial^{2} \phi / \partial z^{2}$ etc. specified at $z=0$ are all even in $r$, it will be found that $g_{1}, g_{2}$ are even and $g_{3}, g_{4}$ odd functions of $t$ and forms like (10) can be used. The majority of problems fall into this category, but there are important exceptions such as the conical punch (where $u_{z}$ is proportional to $r$ ) and problems with Coulomb friction for which $\sigma_{r z}$ is proportional to $\sigma_{z z}$ in some region. In these problems, forms like (10) can only be used if $g_{i}(t)$ is extended into $t<0$ by a definition with the required symmetry.

At the surface $z=0$, the important derivatives of the functions $\phi_{i}$ are given in Table 1.

In certain cases, higher derivatives are required - notably in thermoelastic problems $(1-5,20)$ where heat flux is proportional to $\partial^{3} \phi / \partial z^{3}$. Higher derivatives are most easily obtained by differentiating within the plane $z=0$, making use of the fact that for an axisymmetric harmonic function $f$,

$$
\frac{\partial^{2} f}{\partial z^{2}}=-\frac{1}{r} \frac{\partial}{\partial r} r \frac{\partial f}{\partial r} .
$$

The reader can verify that this result permits the expressions for $\partial^{2} \phi_{i} / \partial z^{2}$ in Table 1 to be obtained from those for $\partial \phi_{i} / \partial r$.

\section{Two-part boundary value problems - examples}

The results of the previous section give a direct solution to any axisymmetric two-part boundary value problem in a single potential function. For example, to find a harmonic function satisfying

$$
\begin{aligned}
& \omega=f(r) ; \quad 0 \leq r \leq a \\
& \omega=0 ; \quad r>a
\end{aligned}
$$

we choose the form $\omega \equiv \partial \phi_{3} / \partial z$. Table 1 shows that (13) is then satisfied identically and (12) gives the Abel equation

$$
-\int_{r}^{a} \frac{g_{3}(t) \mathrm{d} t}{\sqrt{t^{2}-r^{2}}}=f(r) ; \quad 0 \leq r \leq a
$$

to determine the unknown function $g_{3}$. The Abel equations obtained in 
140

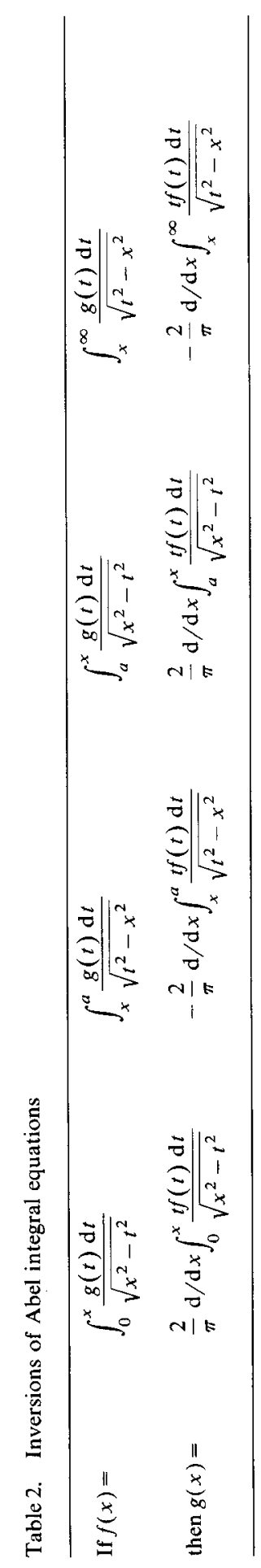


such problems have only four possible forms and the appropriate inversions are given in Table 2 . Notice that these equations can be inverted if and only if their range of application includes the entire range of the unknown function.

If Eq. (12) is replaced by a condition on the derivative

$$
\frac{\partial \omega}{\partial z}=h(r) ; \quad 0 \leq r \leq a
$$

we then find

$$
\frac{1}{r} \mathrm{~d} / \mathrm{d} r \int_{0}^{r} \frac{\operatorname{tg}_{3}(t) \mathrm{d} t}{\sqrt{r^{2}-t^{2}}}=h(r) ; \quad 0 \leq r \leq a
$$

from Table 1. In such cases, we multiply by $r$ and perform an integration in the range $0-r$ or $r-\infty$ to reduce the equation to Abel form. As a second example, if condition (12) is replaced by the radiation condition

$$
\frac{\partial \omega}{\partial z}-H \omega=f(r) ; \quad 0 \leq r \leq a
$$

Table 1 gives immediately the integro-differential equation

$$
\frac{1}{r} \mathrm{~d} / \mathrm{d} r \int_{0}^{r} \frac{t g_{3}(t) \mathrm{d} t}{\sqrt{r^{2}-t^{2}}}+H \int_{r}^{a} \frac{g_{3}(t) \mathrm{d} t}{\sqrt{t^{2}-r^{2}}}=f(r) ; \quad 0 \leq r \leq a
$$

which is Eq. 3.7 of Gladwell et al. [21]. Methods of solving such equations are discussed below.

\subsection{Problems with no zero boundary values}

If non-zero boundary values are prescribed in both parts of the boundary, we decompose the solution into two parts, each of which can be represented in the form of Eq. (5). For example, if

$$
\omega=f_{1}(r) ; \quad 0 \leq r \leq a: \quad \frac{\partial \omega}{\partial z}=f_{2}(r) ; \quad r>a
$$

we write $\omega \equiv \omega_{1}+\omega_{2}$ where $\omega_{1}$ satisfies

$$
\omega_{1}=f_{1}(r) ; \quad 0 \leq r \leq a: \quad \frac{\partial \omega_{1}}{\partial z}=0 ; \quad r>a
$$

and $\omega_{2}$ satisfies

$$
\omega_{2}=0 ; \quad 0 \leq r \leq a: \quad \frac{\partial \omega_{2}}{\partial z}=f_{2}(r) ; \quad r>a
$$

The solution can then be obtained by representing $\omega_{1}, \omega_{2}$ by $\partial \phi_{1} / \partial z$, $\partial \phi_{2} / \partial z$ respectively.

This decomposition is not unique - for example we could define

$$
\omega_{1}=f_{1}(r) ; \quad 0 \leq r \leq a: \quad \omega_{1}=0 ; \quad r>a
$$




$$
\omega_{2}=0 ; \quad 0 \leq r \leq a: \quad \frac{\partial \omega_{2}}{\partial z}=f_{2}(r)-\frac{\partial \omega_{1}}{\partial z} ; \quad r>a
$$

and use $\partial \phi_{3} / \partial z$ for $\omega_{1}$. Notice that in this case $\omega_{1}$ appears in the boundary conditions (23) for $\omega_{2}$ and hence we must solve for $\omega_{1}$ first from (22).

\section{Choice of form-continuity considerations}

Each of the functions $\phi_{i}$ in Table 1 has a zero in $\partial \phi / \partial z$ or $\partial^{2} \phi / \partial z^{2}$ either in $0<r<a$ or in $r>a$. Thus in the above examples it would have been possible to choose $\omega \equiv \partial^{2} \phi_{1} / \partial z^{2}$ instead of $\omega \equiv \partial \phi_{3} / \partial z$.

The choice is best made by examining the requirements of continuity imposed at $r=a, z=0$ by the physical problem. It can be shown that, if $g_{1}(a)$ is bounded, the expressions in Table 1 will define continuous values of $\partial \phi_{i} / \partial z$ through $r=a, z=0$, but $\partial^{2} \phi_{i} / \partial z^{2}$ will be discontinuous unless $g_{i}(a)=0$. Thus, if the function $\omega$ represents a physical quantity like temperature or electrical potential which is required to be continuous, it is appropriate to choose $\omega \equiv \partial \phi_{3} / \partial z$. The alternative choice $\omega=\partial^{2} \phi_{1} / \partial z^{2}$ is not inadmissible, but it would then be necessary to impose the continuity requirement through the auxiliary condition $g_{1}(a)=0$.

In elasticity problems, we require continuity of displacements and this is achieved by expressing $\psi_{1}, \psi_{2}$ in (2) in terms of the $\phi_{i}$ rather than their derivatives. This is why we use the logarithmic form for $\phi_{i}$, in equations (5) in preference to Green's 'point source' form (1), which requires a subsequent stage of partial integration.

In problems involving contact between continuous surfaces - such as the Hertzian problem of a half-space indented by a frictionless paraboloidal punch - the normal traction $\sigma_{z z}$ must also be continuous at the edge of the contact region. The most straightforward treatment of such problems is to use the formulation described above ( $\psi_{i}$ in terms of $\phi_{i}$ ) in which case continuity of normal tractions furnishes an extra condition to determine the radius of the contact region which is not known a priori. However, it is also possible to force the required continuity through the formulation by defining $\partial \psi_{i} / \partial z$ in terms of $\phi_{i}$ in which case the contact radius is prescribed and the rigid body indentation of the punch must be allowed to float. This is essentially the technique used by Segedin [14] in his treatment of the indentation of a half-space by a spherical punch. 


\section{Problems involving several coupled potential functions}

We next consider the application of the method to problems involving several potential functions coupled through the boundary conditions. Typical problems in elasticity are the interface crack and frictional or adhesive punch problems. The procedure is best explained by way of an example for which we use the adhesive indentation of a half-space by a rigid flat-ended cylindrical punch, first solved by Mossakovskii [22].

\subsection{The adhesive punch problem}

The boundary conditions on $z=0$ are

$$
\begin{array}{ll}
u_{z}=d, u_{r}=0 ; & 0 \leq r \leq a \\
\sigma_{z z}=\sigma_{r z}=0 ; & r>a
\end{array}
$$

where $d$ is the depth of indentation and $a$ is the radius of the punch.

To solve this problem, we satisfy (25) by setting $\psi_{1} \equiv \phi_{3}, \psi_{2} \equiv \phi_{1}$ (see Eq. (3) and Table 1), in which case (4) and (24), and Table 1 give the two simultaneous equations

$$
\begin{aligned}
& \frac{(1-2 \nu)}{2 \mu} \int_{r}^{a} \frac{g_{3}(t) \mathrm{d} t}{\sqrt{t^{2}-r^{2}}}-\frac{(1-\nu)}{\mu} \int_{0}^{r} \frac{g_{1}(t) \mathrm{d} t}{\sqrt{r^{2}-t^{2}}}=d ; \quad 0 \leq r \leq a \\
& -\frac{(1-\nu)}{\mu r} \int_{0}^{r} \frac{t g_{3}(t) \mathrm{d} t}{\sqrt{r^{2}-t^{2}}}+\frac{(1-2 \nu)}{2 \mu r}\left[\int_{0}^{a} g_{1}(t) \mathrm{d} t-\int_{r}^{a} \frac{\operatorname{tg}(t) \mathrm{d} t}{\sqrt{t^{2}-r^{2}}}\right]=0 \\
& 0 \leq r \leq a
\end{aligned}
$$

We now treat (26) as an Abel equation for $g_{1}$, carrying the $g_{3}$ integral on to the right hand side and using the inversion rules (Table 2) to obtain

$$
g_{1}(x)=-\frac{2 d \mu}{\pi(1-\nu)}-\frac{2 \beta}{\pi} \int_{0}^{a} \frac{\operatorname{tg}_{3}(t) \mathrm{d} t}{\left(x^{2}-t^{2}\right)} ; \quad 0 \leq x \leq a
$$

where $\beta=(1-2 \nu) / 2(1-\nu)$ is Dundurs constant for this material combination [23].

We note from Table 1 that only four basic forms occur in the surface values of the derivatives $\partial \phi_{i} / \partial r, \partial \phi_{l} / \partial z, \partial^{2} \phi_{i} / \partial z^{2}$. These are the discontinuous functions 


$$
\left.\begin{array}{rlrl}
g_{1}(g, r, a) & =\int_{0}^{r} \frac{g(t) \mathrm{d} t}{\sqrt{r^{2}-t^{2}}} ; & & 0 \leq r \leq a \\
& =\int_{0}^{a} \frac{g(t) \mathrm{d} t}{\sqrt{r^{2}-t^{1}}} ; & & r>a \\
\mathscr{g}_{2}(g, r, a) & =\int_{r}^{a} \frac{g(t) \mathrm{d} t}{\sqrt{t^{2}-r^{2}}} ; & & 0 \leq r \leq a \\
g_{3}(g, r, a) & =0 ; & & r>a \\
& =\int_{a}^{r} \frac{g(t) \mathrm{d} t}{\sqrt{r^{2}-t^{2}}} ; & & r>a \\
\mathscr{g}_{4}(g, r, a) & =\int_{a}^{\infty} \frac{g(t) \mathrm{d} t}{\sqrt{t^{2}-r^{2}}} ; & & 0 \leq r \leq a \\
& =\int_{r}^{\infty} \frac{g(t) \mathrm{d} t}{\sqrt{t^{2}-r^{2}}} ; & & r>a .
\end{array}\right\}
$$

(The expressions for $\partial \phi_{i} / \partial r$ also contain constants in certain cases.) In Table 3 we give the specific inversions for all the combinations of integrals of this type which can occur in the form of Eq. (26). Using this table, Eq. (28) can be written down from case 2 with $i=1, j=2, a=b$ and case 3 with $i=1$, whilst the corresponding inversion of (27) for $g_{3}$ follows from the same two results as

$$
g_{3}(x)=\frac{2 \beta x}{\pi} \int_{0}^{a} \frac{g_{1}(t) \mathrm{d} t}{\left(x^{2}-t^{2}\right)} ; \quad 0 \leq x \leq a .
$$

Notice that $g_{1}$ is even and $g_{3}$ odd as anticipated above (Section 3).

The two Eqs. (28) and (30) can now be solved in a variety of ways, one of which is to substitute (28) into (30) and rearrange the resulting double integral to obtain the Fredholm equation

$$
\begin{aligned}
& \left(1-\beta^{2}\right) g_{3}(x)+\frac{2 \beta^{2}}{\pi^{2}} \int_{0}^{a}\left[x \log \left(\frac{a+t}{a-t}\right)-t \log \left(\frac{a+x}{a-x}\right)\right] \frac{g_{3}(t) \mathrm{d} t}{\left(t^{2}-x^{2}\right)} \\
& =\frac{2 \beta d \mu}{\pi^{2}(1-\nu)} \log \left(\frac{a+x}{a-x}\right) ; \quad 0 \leq x \leq a
\end{aligned}
$$

for $g_{3}$, which can be solved numerically.

Other techniques for treating Eqs. (28), (30) and (31) will be discussed below (Section 7). 
Table 3. (1) Inversions of the equation $\mathscr{q}_{i}(g, r, a)=g_{j}(h, r, b) ; 0 \leq r \leq a$

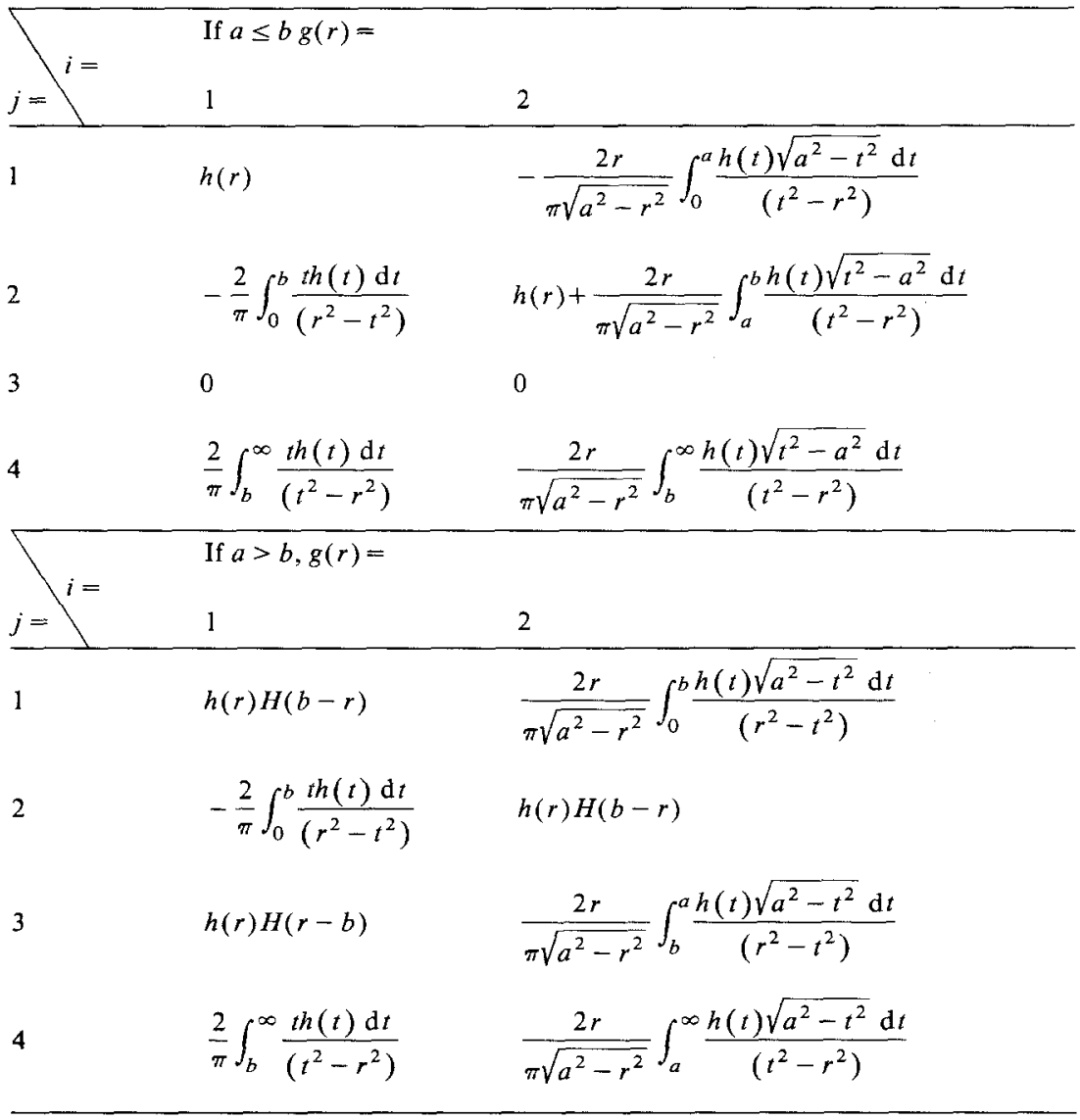

(2) Inversions of the equation $g_{i}(g, r, a)=g_{j}(h, r, b) ; r>a$

$\frac{j={ }_{i=} \text { If } a \geq b, g(r)=}{3} \frac{4}{\frac{2 r}{\pi \sqrt{r^{2}-a^{2}}} \int_{0}^{b} \frac{h(t) \sqrt{a^{2}-t^{2}} \mathrm{~d} t}{\left(r^{2}-t^{2}\right)}} \quad \frac{2 r}{\pi} \int_{0}^{b} \frac{h(t) \mathrm{d} t}{\left(r^{2}-t^{2}\right)}$

2

0

0

3

$$
h(r)+\frac{2 r}{\pi \sqrt{r^{2}-a^{2}}} \int_{b}^{a} \frac{h(t) \sqrt{a^{2}-t^{2}} \mathrm{~d} t}{\left(r^{2}-t^{2}\right)} \quad-\frac{2 r}{\pi} \int_{b}^{\infty} \frac{h(t) \mathrm{d} t}{\left(t^{2}-r^{2}\right)}
$$

4

$$
\frac{2 r}{\pi \sqrt{r^{2}-a^{2}}} \int_{a}^{\infty} \frac{h(t) \sqrt{t^{2}-a^{2}} \mathrm{~d} t}{\left(t^{2}-r^{2}\right)} \quad h(r)
$$


Table 3 (continued)

\begin{tabular}{|c|c|c|}
\hline$j=$ & $\begin{array}{l}\text { If } a<b, g(r)= \\
3\end{array}$ & 4 \\
\hline 1 & $\begin{array}{l}\frac{2 r}{\pi \sqrt{r^{2}-a^{2}}} \int_{0}^{a} \frac{h(t) \sqrt{a^{2}-t^{2}} \mathrm{~d} t}{\left(r^{2}-t^{2}\right)} \\
+h(r) H(b-r)\end{array}$ & $\frac{2 r}{\pi} \int_{0}^{b} \frac{h(t) \mathrm{d} t}{\left(r^{2}-t^{2}\right)}$ \\
\hline 2 . & $\frac{2 r}{\pi \sqrt{r^{2}-a^{2}}} \int_{a}^{b} \frac{h(t) \sqrt{t^{2}-a^{2}} \mathrm{~d} t}{\left(t^{2}-r^{2}\right)}$ & $h(r) H(b-r)$ \\
\hline 3 & $h(r) H(r-b)$ & $-\frac{2 r}{\pi} \int_{b}^{\infty} \frac{h(t) \mathrm{d} t}{\left(t^{2}-r^{2}\right)}$ \\
\hline 4 & $\frac{2 r}{\pi \sqrt{r^{2}-a^{2}}} \int_{b}^{\infty} \frac{h(t) \sqrt{t^{2}-a^{2}} \mathrm{~d} t}{\left(t^{2}-r^{2}\right)}$ & $h(r) H(r-b)$ \\
\hline
\end{tabular}

(3) Inversions of the equation $\mathscr{f}_{i}(g, r, a)=C ; 0 \leq r \leq a$

$$
\begin{array}{ll}
i=1 & g(r)=\frac{2 C}{\pi} \\
i=2 \quad g(r)=\frac{2 C}{\pi} \frac{r}{\sqrt{a^{2}-r^{2}}}
\end{array}
$$

(4) Inversions of the equation $\mathscr{g}_{i}(g, r, a)=C ; r>a$

$i=3$

$$
g(r)=\frac{2 C}{\pi} \frac{r}{\sqrt{a^{2}-r^{2}}}
$$

$i=4 \quad g(r)=0^{*}$

* Note however that the inversion of an equation containing $y_{4}$ is only possible if the right hand side tends to zero as $r \rightarrow \infty$. In certain problems this must be imposed as an auxiliary condition (see e.g. [5])

\subsection{The external interface crack}

This method can still be used if the ranges of definition of the functions $g_{1}, g_{3}$ are different. For example, if instead of the flat rigid punch, we bond a rigid half-space to the elastic half-space in $0 \leq r \leq a, z=0$ and try to separate the bodies by a tensile force along the axis, we should anticipate an annular contact region surrounding the bond, by analogy with the treatment of the penny-shaped interface crack by Keer et al [24]. Assuming this contact region to be frictionless and denoting its outer 
radius by $b$, we obtain the boundary conditions

$$
\begin{array}{ll}
u_{z}=d ; & 0 \leq r \leq b ; \\
u_{r}=0 ; & 0 \leq r \leq a ; \\
\sigma_{z z}=0 ; & r>b ; \\
\sigma_{r z}=0 ; & r>a .
\end{array}
$$

The solution is still obtained as with Eqs. (24) and (25), except that the function $g_{1}$ must now be defined in the range $0 \leq r \leq b$, whilst $g_{3}$ is still defined in $0 \leq r \leq a$. Equations (26) and (27) are now replaced by

$$
\begin{aligned}
& \frac{(1-2 \nu)}{2 \mu} \int_{r}^{a} \frac{g_{3}(t) \mathrm{d} t}{\sqrt{t^{2}-r^{2}}}-\frac{(1-\nu)}{\mu} \int_{0}^{r} \frac{g_{1}(t) \mathrm{d} t}{\sqrt{r^{2}-t^{2}}}=d ; \quad 0 \leq r \leq b \\
& -\frac{(1-\nu)}{\mu r} \int_{0}^{r} \frac{t g_{3}(t) \mathrm{d} t}{\sqrt{r^{2}-t^{2}}}+\frac{(1-2 \nu)}{2 \mu r}\left[\int_{0}^{b} g_{1}(t) \mathrm{d} t-\int_{r}^{b} \frac{b g_{1}(t) \mathrm{d} t}{\sqrt{t^{2}-r^{2}}}\right]=0 \\
& 0 \leq r \leq a
\end{aligned}
$$

where the first integral in (36) is to be interpreted as zero for $r>a$. Table 3 has been constructed to include this more general case $a \neq b$. Notice that Eq. (37) applies only in the range $0 \leq r \leq a$ and hence can be solved for $g_{3}$ but not for $g_{1}$. By contrast, both of Eqs. (26) and (27) could have been solved for either $g_{1}$ or $g_{3}$. Following the same procedure as before we arrive at the Fredholm equation

$$
\begin{aligned}
& \left(1-\beta^{2}\right) g_{3}(x)+\frac{2 \beta^{2}}{\pi^{2}} \int_{0}^{a}\left[x \log \left(\frac{b+t}{b-t}\right)-t \log \left(\frac{b+x}{b-x}\right)\right] \frac{g_{3}(t) \mathrm{d} t}{\left(t^{2}-x^{2}\right)} \\
& =\frac{2 \beta d \mu}{\pi^{2}(1-\nu)} \log \left(\frac{b+x}{b-x}\right) ; \quad 0 \leq x \leq a .
\end{aligned}
$$

In this problem, the radius $b$ defining the extent of the contact region is not known a priori, but must be found by requiring the normal contact traction $\sigma_{z z}$ to be bounded at $r=b$. This requirement gives a side condition which can be used with (38) to obtain a solution for $g_{3}$ and $b$ by iteration as in reference [24]. Notice that the kernels in both Eqs. (31) and (38) are bounded at $x=t$.

It is beyond the scope of this paper to discuss the solution of equations like (38), but we note that the solution might be obtained by direct numerical quadrature [24], by representing the function $g_{3}$ by a truncated power series or Fourier series [25] or a series of orthogonal polynomials [21], or by iteration in the parameter $a / b$ if this is suitably small [16]. 


\subsection{Choice of formulation}

In the above examples, two of the boundary conditions were satisfied identically by the choice of form and the other two furnished coupled Abel integral equations for the two unknown functions $g_{i}$. In all such problems we have to choose which conditions to satisfy identically. If any of the boundary values are zero, the formulation will be simplified by choosing these, but an alternative choice can always be made at the cost of subtracting out one or more two-part solutions as in Section 4.1.

Wherever possible, the choice should be made in such a way that the ranges of the two unknown functions do not overlap, since, if they do, certain of the Abel equation inversions give discontinuous expressions (see, for example, Table 3 case 1 with $i=1, j=1$ and $a>b$ ). This requires that one function be defined in $0 \leq r \leq a$ and the other in $r>b$, where $b>a$. Thus, we try to satisfy identically those conditions which hold in the complementary regions $r>a$ and $0 \leq r \leq b$.

For the external crack problem of Section 6.2, this means satisfying (32) and (35) identically. To do this, we first have to choose a new elasticity solution (Eq. (2)) in order to isolate different single harmonic functions in the expression for $\sigma_{r z}, u_{z}$. Thus, if we write

$$
\psi_{2}=\psi_{3}-\beta \psi_{1}
$$

where $\psi_{3}$ is a new harmonic function, Eqs. (3) and (4) become

$$
\left.\begin{array}{l}
\sigma_{r z}=\frac{\partial^{2} \psi_{1}}{\partial r \partial z} ; \quad \sigma_{z z}=\beta \frac{\partial^{2} \psi_{1}}{\partial z^{2}}-\frac{\partial^{2} \psi_{3}}{\partial z^{2}} ; \\
u_{r}=\left(1-\beta^{2}\right) \frac{(1-\nu)}{\mu} \frac{\partial \psi_{1}}{\partial r}+\frac{(1-2 \nu)}{2 \mu} \frac{\partial \psi_{3}}{\partial r} \\
u_{z}=-\frac{(1-\nu)}{\mu} \frac{\partial \psi_{3}}{\partial z} .
\end{array}\right\}
$$

Equation (35) is satisfied as in Section 6.2 by choosing $\psi_{1} \equiv \phi_{3}$. For (32). following Section 4.1, we subtract an elementary two-part solution from $\psi_{3}$ by writing

$$
\psi_{3} \equiv \psi_{30}+\psi_{31}
$$

where $\psi_{30}$ is defined by

$$
\begin{aligned}
& \frac{\partial \psi_{30}}{\partial z}=-\frac{\mu d}{(1-\nu)} ; \quad 0 \leq r \leq b \\
& \frac{\partial^{2} \psi_{30}}{\partial z^{2}}=0 ; \quad r>b
\end{aligned}
$$

and can easily be found by the methods of Section 4. Equation (32) then 
becomes

$$
\frac{\partial \psi_{31}}{\partial z}=0 ; \quad 0 \leq r \leq b
$$

which is satisfied by choosing $\psi_{3} \equiv \phi_{2}$. It is easily verified that this procedure leads to a pair of equations in two functions $g_{1}, g_{2}$ defined in the ranges $0 \leq r \leq a, r>b$ respectively, as desired.

\section{Extension of ranges}

Some simplification in the algebra and in numerical computation can usually be achieved by using the symmetry of the functions $g_{1}$ to extend the range of integration in the final equations. It is easily shown that if $f(t)$ is even

$$
\int_{0}^{a} \frac{f(t) \mathrm{d} t}{\left(x^{2}-t^{2}\right)}=\frac{1}{2 x} \int_{-a}^{+a} \frac{f(t) \mathrm{d} t}{(x-t)}
$$

whilst if $f(t)$ is odd

$$
\int_{0}^{a} \frac{t f(t) \mathrm{d} t}{\left(x^{2}-t^{2}\right)}=\frac{1}{2} \int_{-a}^{+a} \frac{f(t) \mathrm{d} t}{(x-t)}
$$

For example, using these results in Eq. (38), noting that $g_{3}$ is odd, we obtain the more concise equation

$$
\begin{gathered}
\left(1-\beta^{2}\right) g_{3}(x)+\frac{\beta^{2}}{\pi^{2}} \int_{-a}^{+a} \log \left[\frac{(b+t)}{(b-t)} \cdot \frac{(b-x)}{(b+x)}\right] \frac{g_{3}(t) \mathrm{d} t}{(t-x)} \\
=\frac{2 \beta d \mu}{\pi^{2}(1-\nu)} \log \left(\frac{b+x}{b-x}\right) ; \quad-a \leq x \leq a .
\end{gathered}
$$

Apart from the simplification of the kernel, this equation lends itself to numerical solution using the Chebyshev quadrature which is usually defined in the range -1 to +1 .

The same procedure permits Eqs. (28) and (30) to be reduced to a singular integral equation. Extending the range, we find

$$
\begin{aligned}
& g_{1}(x)=-\frac{\beta}{\pi} \int_{-a}^{+a} \frac{g_{3}(t) \mathrm{d} t}{(x-t)}-\frac{2 d \mu}{\pi(1-\nu)} ; \quad-a \leq x \leq+a, \\
& g_{3}(x)=\frac{\beta}{\pi} \int_{-a}^{+a} \frac{g_{1}(t) \mathrm{d} t}{(x-t)} ; \quad-a \leq x \leq+a,
\end{aligned}
$$

and hence

$$
g(x)-\frac{\beta}{\pi i} \int_{-a}^{+a} \frac{g(t) \mathrm{d} t}{(t-x)}=\frac{-2 d \mu}{\pi(1-\nu)} ; \quad-a \leq x \leq+a,
$$


where

$$
g(x) \equiv g_{1}(x)+i g_{3}(x) .
$$

The reduction of Mossakovskii's problem to the solution of Eq. (50) was given by Gladwell [9], Section 10.4.

If the forms II, IV of Table 1 are used, the resulting equations will generally involve integrals in the range $a<x<\infty$. In this case, the most convenient procedure is to make the change of variable $y=1 / x$ to convert the range to $0<y<1 / a$ after which the range can generally be extended to $-1 / a<x<+1 / a$ using (45) and (46). If the problem is well formulated, the behavior of the functions $g_{i}$ as $x \rightarrow \infty$ will permit this change of variable without introducing singularities at zero.

We note that the forms such as Eq. (10) lead directly to equations in the extended range, but further algebraic manipulation is sometimes then required to express the final results in the most concise form.

\section{Problems involving annular regions}

We now consider the application of the method to problems in which boundary values are specified over an annular region, the classical example being the three part problem defined by

$$
\begin{array}{lc}
\partial \omega / \partial z=0 ; & 0 \leq r \leq a \\
\omega=f(r) ; & a<r \leq b \\
\partial \omega / \partial z=0 ; & r>b
\end{array}
$$

and solved by Collins [16]. The solution can be used with Eqs. (2)-(4) for the indentation of an elastic half-space by an annular cylindrical punch.

The solution technique is to express $\omega$ as the sum of a number of components satisfying two part boundary conditions and hence expressible in the forms of Eq. (5). For example, we can write $\omega \equiv \omega_{1}+\omega_{2}$ where

$$
\begin{array}{ll}
\omega_{1}=f(r) ; \quad 0 \leq r \leq b & \\
\partial \omega_{1} / \partial z=-\partial \omega_{2} / \partial z ; \quad & r>b \\
\partial \omega_{2} / \partial z=-\partial \omega_{1} / \partial z ; \quad 0 \leq r \leq a \\
\omega_{2}=0 ; \quad r>a .
\end{array}
$$

It is easily verified that this decomposition satisfies Eqs. (52)-(54) and the function $\omega_{2}$ can be represented by $\partial \phi_{3} / \partial z$ (see Table 1). However, the prescribed values of $\omega_{1}$ are non-zero for all $r$ and a further decomposition is necessary as in Section 4.1. In fact Eqs. (41) and (42) are of the same form as (19) and can be treated in the same way.

When the boundary conditions are written in terms of the expressions of Table 1, it will be found that Eqs. (56) and (57) give two coupled Abel 
equations for two unknown functions $g_{i}$ in the ranges $r>b$, or $0 \leq r \leq a$ respectively. The solution and reduction to a single Fredholm equation then proceeds as in Section 6.

Notice that the annular condition (53) is met by 'overlapping' the two part conditions (55) and (58) on $\omega_{1}, \omega_{2}$, respectively. In effect, $\omega_{1}$ 'extends' (53) to zero and $\omega_{2}$ is chosen to relax this overprescription of the problem in $0 \leq r \leq a$, whilst leaving $\omega$ unchanged in $a<r \leq b$. For more complex examples of this procedure, the reader is referred to references [3], [4], [5] and [26]. A difficulty arises if the function $f(r)$ in Eq. (53) is not defined in $0 \leq r \leq a$ or if it has undesirable singularities in this range (possibly at $r=0$ ). Collins treats this problem by decomposing $f(r)$ into two series - one in powers of $r$ and one in powers of $1 / r$. The latter is then treated by a process similar to that described above except that (53) is now extended into the range $a<r<\infty$ with a corresponding change in the range of definition of $\omega_{2}$.

Alternatively, we can avoid this difficulty by using any convenient continuation of $f(r)$ into $0<r<a$ at the cost of somewhat greater complexity in the subsequent manipulations. However, the numerical solution of the final equation will generally be more convergent if we choose a continuation which in continuous with $f(r)$ at least to the first derivative at $r=a$.

\section{Total load and stress intensity factors}

In the solution of elastic contact and crack problems, the quantities of most practical interest are the total normal load transmitted at the interface and the stress intensity factors at the crack tip.

The total normal load transmitted through the region $0 \leq r \leq a, z=0$

Table 4. Expressions for calculating total load

\begin{tabular}{llc}
\hline & $\int_{0}^{a} r \frac{\partial^{2} \phi_{i}}{\partial z^{2}} \mathrm{~d} r$ & $\int_{a}^{\infty} r \frac{\partial^{2} \phi_{i}}{\partial z^{2}} \mathrm{~d} r$ \\
\hline 1 & $-\int_{0}^{a} g_{1}(t) \mathrm{d} t$ & 0 \\
3 & $\int_{a}^{\infty}\left\{\frac{t}{\sqrt{t^{2}-a^{2}}}-1\right\} g_{2}(t) \mathrm{d} t$ & $-\int_{a}^{\infty} \frac{\operatorname{tg}_{2}(t) \mathrm{d} t}{\sqrt{t^{2}-a^{2}}}$ \\
& $\int_{0}^{a} \frac{\operatorname{tg}_{3}(t) \mathrm{d} t}{\sqrt{a^{2}-t^{2}}}$ & $-\int_{0}^{a} \frac{\operatorname{tg}_{3}(t) \mathrm{d} t}{\sqrt{a^{2}-t^{2}}}$ \\
& 0 & $-L_{t \rightarrow \infty}\left(\frac{\pi t g_{4}(t)}{2}\right)$
\end{tabular}


is

$$
P=2 \pi \int_{0}^{a} r \sigma_{z z} \mathrm{~d} r
$$

and this will generally involve integrals of the form $\int_{0}^{a} r\left(\partial^{2} \phi_{i} / \partial z^{2}\right) \mathrm{d} r$. These integrals can be expressed in terms of $g_{i}$ by substituting from Table 1 and reversing the order of integration and the results are given in Table 4. Also in Table 4 we give the expressions $\int_{a}^{\infty} r\left(\partial^{2} \phi_{i} / \partial z^{2}\right) \mathrm{d} r$ which will appear in computations of the load transmitted through the region $r>a, z=0$.

Notice that sum of the two expressions for $\phi_{3}$ is zero, indicating that this representation does not permit any net normal load to be transmitted across the plane $z=0$ (and hence across any parallel plane). This result is a consequence of the boundary condition $\phi_{3}=0, r>a$ (see Table 1). It is shown in (21) that the same result holds with the weaker condition

$$
h \phi_{3}+\frac{\partial \phi_{3}}{\partial z}=0 ; \quad z=0, r>a
$$

for any $h>0$.

Stress intensity factors in mode I $\left(\sigma_{z z}\right)$ and mode II $\left(\sigma_{r z}\right)$ can arise from square root singularities in $\partial^{2} \phi_{i} / \partial z^{2}$ and $\partial^{2} \phi_{i} / \partial r \partial z$ respectively. The asymptotic behavior of these derivatives near $r=a$ can be examined by integrating by parts the expressions from Table 1. For example, we find

$$
\begin{aligned}
\frac{\partial^{2} \phi_{3}}{\partial z^{2}} & =-\frac{g_{3}(0)}{r}-\int_{0}^{r} \frac{g_{3}^{\prime}(t) \mathrm{d} t}{\sqrt{r^{2}-t^{2}}} ; \quad 0 \leq r \leq a \\
& =-\frac{g_{3}(0)}{r}+\frac{g_{3}(a)}{\sqrt{r^{2}-a^{2}}}-\int_{0}^{a} \frac{g_{3}^{\prime}(t) \mathrm{d} t}{\sqrt{r^{2}-t^{2}}} ; \quad r>a
\end{aligned}
$$

from which

$$
\underset{r \rightarrow a^{+}}{L t} \sqrt{r^{2}-a^{2}} \frac{\partial^{2} \phi_{3}}{\partial z^{2}}=g_{3}(a) .
$$

We note that the integral terms in Eqs. (61) and (62) will also be singular if $g_{3}(t)$ contains a term which behaves like $(a-t)^{\alpha}$ near $t=a$, where $0<\alpha \leq 1 / 2$. However, this singularity will be dominated by the square root term in Eq. (62) unless $g_{3}(a)=0$. In particular, if $\alpha=1 / 2$, both Eqs. (61) and (62) are logarithmically singular at $r=a$. This type of singularity occurs in the heat flux at the transition between perfect and imperfect contact $[2,5,10]$. By contrast, if $g_{3}$ has a Taylor series expansion at $r=a$ with non-zero $g_{3}(a), \partial^{2} \phi_{3} / \partial z^{2}$ is square root singular in $r \rightarrow a^{+}$, but bounded in $r \rightarrow a^{-}$.

Stress intensity factors for both $\partial^{2} \phi_{i} / \partial z^{2}$ and $\partial^{2} \phi_{i} / \partial r \partial z$ are given for the four forms $\phi_{i}$ in Table 5. Notice that each function is singular in 
$\partial^{2} \phi_{i} / \partial z^{2}$ on one side of $r=a$ and in $\partial^{2} \phi_{i} / \partial r \partial z$ on the other. The implication of this result will depend upon the solution of the elasticity equation which is used. If the same function $\psi_{i}$ appears in the expression for both $\sigma_{r z}$ and $\sigma_{z z}$ - as with $\psi_{1}$ in the solution of Eqs. (40) and (41) stress intensity factors in modes I, II will tend to occur on opposite sides of $r=a$ and be related in magnitude. By contrast, the function used to represent $\psi_{1}$ in Eqs. (2)-(4) will not introduce a stress intensity factor in mode $\mathrm{I}$, even if it has an appropriate singularity in $\partial^{2} \phi_{i} / \partial z^{2}$.

With this in mind, it is generally desirable to use a solution which isolates the discontinuous behaviour of the tractions at each transition in a different function. For example, the solution (40) and (41)) is well suited to the external crack problem, because the boundary conditions (43) ensure that the function $\psi_{3}$ is continuous through the crack tip, $r=a$. Thus, the singularities associated with the crack tip are isolated in $\psi_{1}$ and comprise stress intensity factors of $-\beta g_{1}(a)$ in compression in $r \rightarrow a^{+}$(since $a<r \leq b$ is a region of contact) and $-g_{1}(a)$ in shear in $r \rightarrow a^{-}$. This relationship between stress intensity factors in modes I and II on opposite sides of the crack tip is typical of interface crack problems with a contact region at the crack tip. In this problem there is also a discontinuity in the slope of the contact tractions at $r=b$ and this is isolated in the function $\psi_{3}$.

\section{Thermoelastic problems}

The method is easily adapted to the solution of boundary value problems in steady-state thermoelasticity by the inclusion of a third potential function related to temperature. A suitable solution is obtained by adding $2 \delta \mathbf{k}\left(\partial \psi_{3} / \partial z\right)-\delta \nabla \psi_{3}$ into the right hand side of Eq. (2), corresponding to the temperature field

$$
T=\frac{1}{k} \frac{\partial^{2} \psi_{3}}{\partial z^{2}}
$$

where

$$
\delta=\alpha(1+\nu) / k
$$

is the distortivity of the material and $\alpha, k$ are respectively the coefficient of thermal expansion and thermal conductivity. This solution is due to Williams [27].

With the additional function $\psi_{3}$, Eqs. (3) are unchanged, and Eqs. (4) become

$$
\begin{aligned}
& u_{r}=\frac{(1-\nu)}{\mu} \frac{\partial \psi_{1}}{\partial r}+\frac{(1-2 \nu)}{2 \mu} \frac{\partial \psi_{2}}{\partial r}-\delta \frac{\partial \psi_{3}}{\partial r} \\
& u_{z}=-\frac{(1-2 \nu)}{2 \mu} \frac{\partial \psi_{1}}{\partial z}-\frac{(1-\nu)}{\mu} \frac{\partial \psi_{2}}{\partial z}+\delta \frac{\partial \psi_{3}}{\partial z}
\end{aligned}
$$




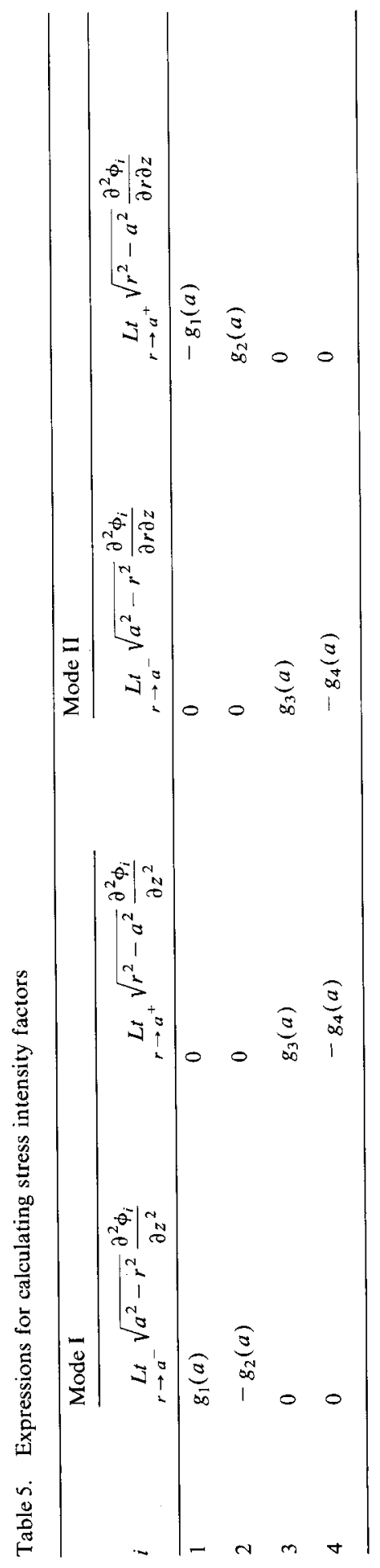


Also, the heat flux

$$
q_{z}=-\frac{k \partial T}{\partial z}=-\frac{\partial^{3} \psi_{3}}{\partial z^{3}}
$$

from Eq. (64).

Steady-state heat conduction in the half-space is formally equivalent to the potential problems associated with frictionless contact, as can be seen by comparing Eqs. (64) and (68) with (3) and (4) when $\psi_{1} \equiv 0$. Notice, however, that the comparable quantities in this analogy are $\partial \psi_{2} / \partial z$ (normal displacement) and $\partial^{2} \psi_{3} / \partial z^{2}$ (temperature). Both of these quantities are required to be physically continuous at $r=a, z=0$ and following the arguments of Section 5 we should represent $\partial \psi_{3} / \partial z \equiv$ $\phi_{i}$. However, in order to do this we have to obtain further partial integrals of the expressions in Table 1 and these are complicated and cumbersome to use. A more algebraically convenient approach is to choose $\psi_{3} \equiv \psi_{i}$, in which case the physical requirement of continuity of temperature must be imposed as an extra condition. It is clear from the discussion of stress intensity factors and Table 5 that this condition will always require $g_{i}(a)=0$, though there may be supplementary conditions if $g_{i}^{\prime}$ is unbounded at $r=a$. For examples of the use of these conditions, the reader is referred to references [1]-[5].

A further difficulty with the representation $\psi_{3} \equiv \phi_{i}$ is that it is liable to lead to unbounded integrals if the problem is such that the net heat flow across the plane $z=0$ is non-zero. This arises from the fact that a point heat source gives a temperature which is inverse with $r$ and hence displacements which are logarithmically unbounded at infinity (28). The most important class of problems exhibiting this behavior is the indentation of a half-space by a heated punch, the non-contact region surrounding the punch being assumed insulated $\left(q_{z}=0\right)$. However, we note from Section 9 that the total heat flow across the plane will be constrained to be zero if the external boundary condition is $T=0$ or $\partial T / \partial z+h T=0$ (i.e. if there is radiation into a medium at zero). This question is further discussed by Gladwell et al. [21]. For the same reason, the problem of a uniform flow of heat obstructed by an internal crack automatically satisfies the condition of zero total heat flow, provided it is formulated as a perturbation of the solution for an unflawed solid. By contrast there will be a non-zero heat flux if heat is generated at the crack face or if the crack is external.

For problems with a non-zero total heat flow it is essential to use a representation which can accommodate logarithmically unbounded displacements at infinity. This can be done by subtracting out an arbitrary multiple of a classical solution with the appropriate behavior at infinity. For example, we can write

$$
\begin{aligned}
\psi_{3} \equiv & \phi_{i}+A\left\{(z+i a) \log \left[\left(r^{2}+(z+i a)^{2}\right)^{1 / 2}+(z+i a)\right]\right. \\
& \left.-\left(r^{2}+(z+i a)^{2}\right)^{1 / 2}\right\}
\end{aligned}
$$


where $A$ is an arbitrary constant which will be determined from the conditions imposing continuity of temperature.

\section{Acknowledgement}

The author gratefully acknowledges support from the U.S. Army under contract DAAG 29-82-K-0001.

\section{References}

1. Barber JR (1978) Contact problems involving a cooled punch. J Elasticity, 8:409-423

2. Barber JR (1982) Indentation of an elastic half-space by a cooled flat punch. Q J Mech Appl Math 35:141-154

3. Barber JR and Comninou M (1982) The external axisymmetric interface crack with heat flow. Q J Mech Appl Math 35:403-417

4. Martin-Moran CJ, Barber JR and Comninou M, The penny-shaped interface crack with heat flow: I. Perfect contact. J Appl Mech (in press)

5. Barber JR and Comninou M, The penny-shaped interface crack with heat flow: II. Imperfect contact. J Appl Mech (under review)

6. Sneddon IN (1966) Mixed boundary value problems in potential theory. North Holland, Amsterdam

7. Sneddon IN (1969) Crack problems in the classical theory of elasticity. John Wiley, New York

8. Keer LM (1964) A class of non-symmetrical punch and crack problems. Q J Mech Appl Math 17:423-436.

9. Gladwell GML (1980) Contact problems in the classical theory of elasticity. Sijthoff \& Noordhoff, Alphen aan den Rijn: 507-509

10. Love AEH (1939) Boussinesq's problem for a rigid cone. Q J Math 10:161-175

11. Green AE (1949) On Boussinesq's problem and penny-shaped cracks. Proc Cambridge Phil Soc 45:251-257

12. Copson ET (1947) On the problem of the electrified disc. Proc Edinburgh Math Soc 8:14-19

13. Green AE and Zerna W (1954) Theoretical elasticity. Clarendon Press, Oxford

14. Segedin CM (1957) The relation between load and penetration for a spherical punch. Mathematika 4:156-161

15. Maw N, Barber JR and Fawcett JN (1976) The oblique impact of elastic spheres. Wear 38:101-114

16. Collins WD (1963) On the solution of some axisymmetric boundary value problems by means of integral equations: IV. Potential problems for a circular annulus. Proc Edinburgh Math Soc 13:235-246

17. Collins WD (1959) On the solution of some axisymmetric boundary value problems by means of integral equations, II: Further problems for a circular disc and a spherical cap. Mathematika, 6:120-133

18. Keer LM (1965) A note on the solution for two asymmetric boundary value problems. Int J Solids Structures, 1:257-264

19. Keer LM (1967) Mixed boundary-value problems for an elastic half-space. Proc Cambridge Phil Soc 63:1379-1386

20. Shail $R$ (1968) Some steady-state thermoelastic stress distributions in the vicinity of an external crack in an infinite solid. Int J Engng Sci 6:685-694 
21. Gladwell GML, Barber JR and Olesiak Z, Thermal problems with radiation boundary conditions, Q J Mech Appl Math (under review)

22. Mossakovskii VI (1963) Compression of elastic bodies under conditions of adhesion (Axisymmetric case). J Appl Math Mech 27:630-643

23. Dundurs J (1969) Discussion on Edge-bonded dissimilar orthogonal elastic wedges under normal and shear loading, J Appl Mech 36:650-652

24. Keer LM, Chen SH and Comninou M (1978) The interface penny-shaped crack reconsidered, Int J Engng Sci 16:765-772

25. Barber JR (1976) Some thermoelastic contact problems involving frictional heating, Q J Mech Appl Math 29:1-13

26. Barber JR (1983) A four-part boundary value problem in elasticity - indentation by a discontinuously concave punch. App Sci Res 40: 159-167

27. Williams WE (1961) A solution of the steady-state thermoelastic equations, Z Angew Math Phys 12:452-455

28. Barber JR (1971) The solution of heated punch problems by point source methods, Int J Engng Sci 9:1165-1170 